\title{
Blind Equalization using Constant Modulus Algorithm and Multi-Modulus Algorithm in Wireless Communication Systems
}

\author{
Ram Babu. T \\ Electronics and Communication \\ Department \\ Rao and Naidu Engineering College, \\ Ongole 523001 A.P \\ INDIA.
}

\author{
Dr.P.Rajesh Kumar \\ Associate Professor \\ Electronics and Communication \\ Department \\ Andhra University College of \\ Engineering \\ Visakhapatnam. 530003 A.P., INDIA
}

\begin{abstract}
It is a method of adaptive multi-modulus equalization for an equalizer. A cost from a cost function is calculated according to a constant modulus algorithm (CMA). Equalizer coefficients are updated according to the cost, and modulus of each region is determined for a multi-modulus algorithm (MMA). The equalizer is switched to use the MMA when the cost reaches a first threshold. The MMA comprises several stages determined by thresholds, and the number of regions increases in every stage. The cost is calculated according to the MMA and modulus of each region. The equalizer coefficients are updated according to the cost, and modulus of each region is determined for the subsequent stage of the MMA. The equalizer is switched to the subsequent stage of the MMA when the MMA cost function output reaches the threshold corresponding to the current stage. The steps are repeated until the cost reaches a preset value. Then, the number of regions and equalizer coefficients are fixed to equalize the input signal.
\end{abstract}

\section{Keywords}

Equalization, CMA, MMA, cost function.

\section{INTRODUCTION}

There are many potential exciting applications of blind signal processing in science and technology, especially in wireless communication, noninvasive medical diagnosis, geophysical exploration, and image enhancement and recognition. Blind methods are of great importance in digital signal communication systems as they allow channel equalization at the receiver without the use of training signals. The topic of blind equalization of linear time invariant (LTI) channels, both SIMO and MIMO, has drawn considerable attention over the past years and several algorithms have been developed. One of the practical problems in digital communications is inter-symbol interference (ISI), which causes a given transmitted symbol to be distorted by other transmitted symbols. The ISI is imposed on the transmitted signal due to the band limiting effect of the practical channel and also due to the multi-path effects (echo) of the channel. One of the most commonly used techniques to counter the channel distortion (ISI) is linear channel equalization. The equalizer is a linear filter that provides an approximate inverse of the channel response. Since it is common for the channel characteristics to be unknown or to change over time, the preferred embodiment of the equalizer is a structure that is adaptive in nature. Conventional equalization techniques employ a pre-assigned time slot (periodic for the time-varying situation) during which training signal, known in advance by the receiver, is transmitted. In the receiver the equalizer coefficients are then changed or adapted by using some adaptive algorithm (e.g. LMS, RLS, etc.) so that the output of the equalizer closely matches the training sequence. However, inclusion of this training sequence with the transmitted information adds an overhead and thus reduces the throughput of the system. Therefore, to reduce the system overhead, adaptation schemes are preferred that do not require training, i.e., blind adaptation schemes. In blind equalization, instead of using the training sequence, one or more properties of the transmitted signal are used to estimate the inverse of the channel.

The problem with blind adaptation techniques is their poor convergence property compared to traditional techniques using training sequences. Generally a gradient descent based algorithm is used with the blind adaptation schemes. The most commonly used gradient descent based blind adaptation algorithm is the Constant Modulus Algorithm (CMA). CMA exploits the constant modularity of the transmitted signal for adapting the parameters of an equalizer. The counterpart of CMA is the Least Mean Square (LMS) algorithm that uses a training sequence for the adaptation process. Due to the knowledge of the transmitted sequence, the LMS algorithm, if convergent, will always converge to the global minimum. Moreover, for a particular delay in the overall system, the LMS cost function is quadratic and provides only a single global minimum in the cost surface. Therefore, irrespective of the initialization, the LMS algorithm will converge to the global minimum. If the initialization is such that adaptation takes place in a major eigen space only, the convergence is fast. For CMA based schemes, where the receiver does not know the transmitted sequence, any sequence with a constant phase offset with the input sequence may be considered to be the right sequence at the receiver since the phase shift does not change the constant modularity property of a signal. Due to this reason, unlike the LMS cost surface, the Constant Modulus (CM) cost surface will have multiple minima. Each of the minima will correspond to a unique phase shift. For a length $\mathrm{N}$ equalizer, the number of minima of the CM cost surface is $\mathrm{N} 2$; in other words, there are $\mathrm{N} 2$ different phase shifts for which there exist solutions in the CM sense. For most practical purposes, all of the N2 solutions are not equally acceptable. If the source sequence is a differentially encoded M-ary PSK signal, for any transmitted sequence, $\mathrm{M}$ different sequences will be acceptable at the receiver. Therefore, out of the N2 solutions only M will be acceptable and the minima corresponding to these acceptable solutions are called global minima. All other minima are called local minima, even though the cost at all minima is the same if the equalizer is not under-modeled. The initialization of the equalizer determines the minimum point on the cost surface whereto CMA will force the equalizer to converge. Therefore, depending on its initialization, an equalizer employing CMA may converge to a local or a global minimum. Another problem with the CMA algorithm is that the convergence rate is much slower than the convergence rate of any gradient descent algorithm using a training sequence. 


\section{BLIND CHANNEL EQUALIZATION AND TYPES}

The field of blind channel equalization has been existence for a little over twenty years. Research during this time has centered on developing new algorithms and formulating a theoretical justification for these algorithms. Blind channel equalization is also known as a self-recovering equalization.. The objective of blind equalization is to recover the unknown input sequence to the unknown channel based solely on the probabilistic and statistical properties of the input sequence. The receiver can synchronize to the received signal and to adjust the equalizer without the training sequence. The term blind is used in this equalizer because it performs the equalization on the data without a reference signal. Instead, the blind equalizer relies on knowledge of the signal structure and its statistic to perform the equalization.

1. Blind signal is the unknown signal which would be identified in output signal with accommodated noise signal at receiver.

2. Channel equalization uses the idea $\&$ knowledge of training sequences for channel estimation where as Blind channel equalization doesn't utilizes the characteristics of training sequences for frequency and impulse response analysis of channel.

3. Blind Channel Equalization differs from channel equalization and without knowing the channel characteristics like transfer function \& SNR it efficiently estimate the channel and reduces the ISI by blind signal separation at receiver side by suppressing noise in the received signal.

\section{CMA - CONSTANT MODULUS ALGORITHM}

In digital communication, equalizer was designed to compensate the channel distortions, through a process known as equalization. There are two types of equalization which are: 1) Trained equalization, 2) Blind (self-recovering) Equalization.

Blind equalization finds important application in data

communication s ys t e ms. In data communications, digital signals are generated and transmitted by the sender through an analog channel to the receiver. Linear channel distortionas a result off 1 imited channel bandwidth, multipath and fading is often the most serious distortion in digital communication system.

Blind equalization improves system bandwidth efficient by avoiding the use of training sequence. The linear channel distortion, known as the Inter-symbol interference (ISI), can severely corrupt the transmitted signal and make it difficult for the receiver to directly recover the transmitted data. Channel equalization and identification has proven to be an effective means to compensate the linear distortion by removing much of the ISI.

\section{Channel Equalization:}

A typical communication system design involves first passing the signal to be transmitted through a whitening filter to reduce redundancy or correlation and then transmitting the resultant whitened signal. At the receiver, the recorded signal is passed through the inverse whitening filter and the original signal is thus restored. However, the channel will affect the transmitted signal because of a) Channel noise b)
Channel dispersion leading to inter symbol interference. For example, by reflection of the transmitted signal from various objects such as buildings in the transmission path, leading to echoes of the transmitted signal appearing in the receiver. Therefore, it is necessary to pass the received signal through a so called

equalizing filter to undo the dispersion effect as shown in figure 2 below. Equalization compensates for Inter symbol Interference (ISI) created by multi path within time dispersive channel

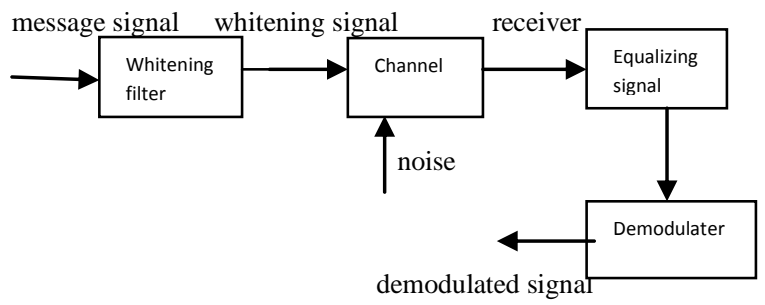

Fig 1: Block diagram of Channel Equalization

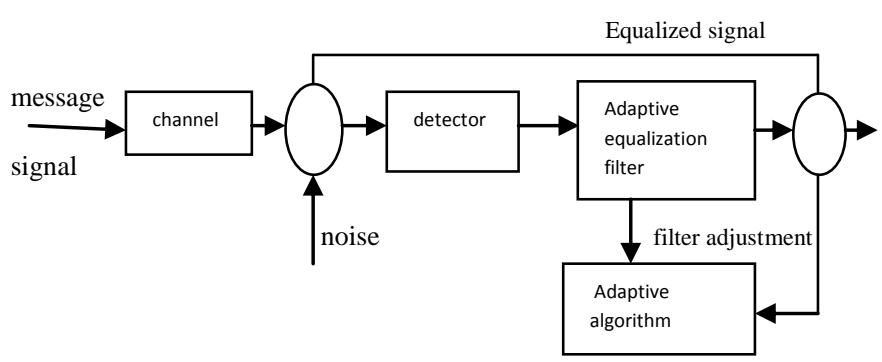

Fig 2 : Adaptive Equalization

\section{a. Blind Channel Equalization:}

The field of blind channel equalization has been existence for a little over twenty years. Research during this time has centered on developing new algorithms and formulating a theoretical justification for these algorithms. Blind channel equalization is also known as a self-recovering equalization. The objective of blind equalization is to recover the unknown input sequence to the unknown channel based solely on the probabilistic and statistical properties of the input sequence. The receiver can synchronize to the received signal and to adjust the equalizer without the training sequence. The term blind is used in this equalizer because it performs the equalization on the data without a reference signal. Instead, the blind equalizer relies on knowledge of the signal structure and its statistic to perform the equalization.

A natural question from for direct adaptive equalization with training is, "How can we adapt our filter F, without the use of a training signal?". Figure 2 shows such a system. There has been extensive research on this subject for single user applications as well as multi-user applications. The Constant Modulus Algorithm is one such algorithm employed for the blind adaptation problem. 


\section{MMA - MULTI MODULUS ALGORITHM}

The present invention relates to a method of blind equalization in a receiver and particularly to an adaptive multi-modulus equalization method. Signals transmitted through a real voiceband channel suffer from non-ideal channel characteristics such as Additive White Gaussian Noise (AWGN), Inter Symbol Interference, fading, and phase distortion. These non- ideal channel characteristics seriously degrade and distort the original signals. When a signal passes through a particular channel, the receiver can model the effect of the channel as a filter with a transfer function $\mathrm{H}(\mathrm{z})$. To overcome the non-idea channel characteristics, the receiver thus deigns an adaptive filter with a transfer function $\mathrm{H}-1(\mathrm{z}) . \mathrm{H}(\mathrm{z})$ is the inverse transfer function of $\mathrm{H}(\mathrm{z})$, and multiplying $\mathrm{H}-1$ (z) to the signal with channel effect recovers the original signal by canceling $\mathrm{H}(\mathrm{z})$. The expected response of combining the adaptive filter with the real voiceband channel is an impulse response in the time domain, and constant over all frequency range in the frequency domain. The adaptive filter described is referred to as an equalizer. An ideal equalizer recovers signals passed through the real voice-band channel, and completely removes the channel effect.

The coefficients of the equalizer, also referred as tap weights, determine the transfer function of the equalizer. The tap weights need to be adjusted and updated frequently to minimize error at the output of the equalizer. This error is effectively a measure of the difference between the actual output of the equalizer and the expected output. Generally speaking, there are two ways of acquiring new tap weights for the equalizer. One is to transmit a training sequence known by both transmitter and receiver at the beginning of the communication. The receiver then detects the impulse response of the channel from the training sequence, and obtains the tap weights by computing the inverse transfer function of the channel. The other way is to predetermine an initial value for each of the tap weights, and design a cost function according to the characteristics of the received signal. The tap weights are continually adjusted by reducing the cost of the cost function until the error is minimized (i.e. until the equalizer converges). Equalizers implementing the second technique described above are referred to as "blind equalizers".

The channel effect varies, and an adaptive equalizer with adjustable transfer function is required to adapt any instantaneous change in the channel effect. The characteristics of the channel change slowly with temperature, movement of the receiver, and many other environmental factors. The adaptive equalizer continuously updates its transfer function by adjusting the tap weights to compensate for current channel effect. Blind equalization is considered more effective than non-blind equalization due to its ability to update the tap weights at any time without waiting for a training sequence.

Constant modulus algorithm (CMA) is a well- known technique used in blind equalization. The CMA algorithm converges an equalized signal on a constellation diagram with constellation points scattered evenly over several concentric circles. An attribute of the CMA algorithm is that the blind equalizer does require the number of valid coordinates on the constellation diagram.
The CMA algorithm defines a cost function to estimate channel noise in a received signal. The higher the output (cost) of the cost function, the larger the channel noise in the received signal. The equalizer first calculates an equalized signal by adding the products of the received signal and the tap weights. After obtaining the equalized signal, the cost function calculates the cost of the equalized signal. The cost indicates the noise level of the received signal, and this cost is used to adjust the tap weights of the equalizer. The equalizer then calculates a new equalized signal using the updated tap weights, and obtains a new cost from the new equalized signal. The cost of the cost function is expected to be reduced by repeating the above processes. The lower the cost, the lower the noise in the received signal.

The cost function of $\mathrm{pth} i$ order ( $\mathrm{p}$ is an integer greater than zero) is given by:

$$
D^{(p)}=E\left[\left(\left|Z_{n}\right|^{p}-R_{p}\right)^{2}\right]
$$

Where $\mathrm{Z}$ is the output (equalized signal) of the equalizer, and $\mathrm{R}$ is a positive constant. The equation used to calculate the least-mean-squares (LMS) error can also be the cost function for adjusting the tap weights. The LMS error is given by:

$$
\text { Error=E[( } \left.\left.Z_{n}-a_{n}\right)^{2}\right]
$$

While there is a great similarity between equation (1) and equation (2), an expected output an of the equalizer, the original signal without channel noise, must be known in advance when using the LMS cost function. The LMS cost function is therefore not suitable for a monotonic demodulating system, as the original signal is unknown to the receiver.

An advantage of using the pth order cost function is the ability to achieve convergence without knowing the original signal in advance. By using the pth order cost function, $\mathrm{Rp}$ is assumed to be the ideal output of the equalizer. However, pth order cost function minimizes the difference between $\mathrm{Rp}$ and $|\mathrm{Zn}| \mathrm{p}$, which takes longer to achieve convergence than the LMS technique. Another drawback to pth order cost function is that carrier phase distortion cannot be recovered.

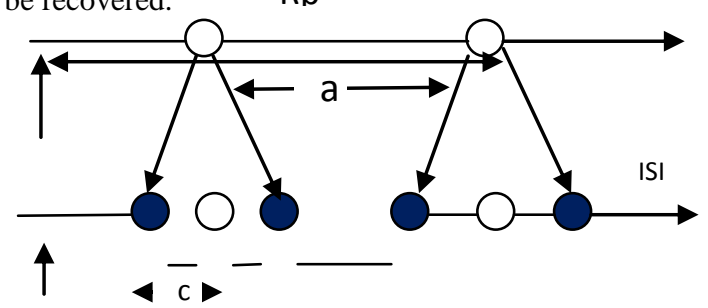

Figure 3 ISI effect on a signal.

FIG. 3 is a diagram illustrating the ISI effect on a signal. ISI is considered to cause the most serious distortion to the signal compared to other types of channel noise. Locations of white dots therein represent coordinates of an original signal without ISI interference on a constellation diagram. The original signal interferes with ISI when transmitting on a physical channel, causing the coordinates of the received signal to shift locations of black dots on the constellation diagram. 


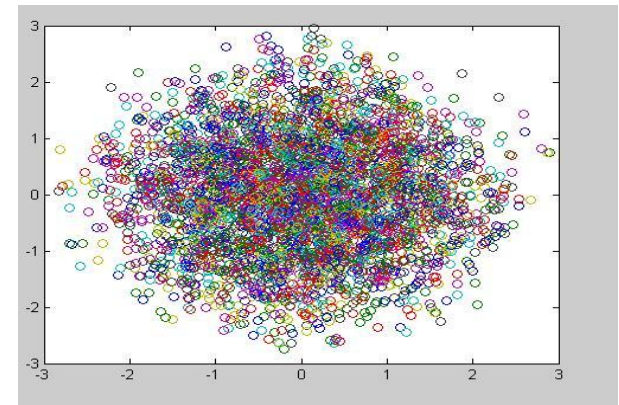

Figure 4. constellation diagram illustrating an output of a CMA equalizer when receiving QAM signal

As shown in the figure 4, the black dots represent coordinates of the signal with ISI interference. The cost, that is the output of the cost function, of the original signal is $2 \mathrm{a} 2$, whereas the cost of the signal with ISI is $2 a 2+4 c 2$. The difference of $4 \mathrm{c} 2$ indicates that the cost of the signal with ISI is greater than that of the original signal. Adjusting the coefficients (tap weights) of the equalizer can reduce costs and cancel the effects of ISI interference. The steepest gradient descent method is a possible solution for adjusting the tap weights to minimize the cost of the cost function. Equations (3), (4), and (5) show the formula for adjusting the tap weights.

$$
\begin{gathered}
C_{n+1}=C_{n}-\lambda_{p}\left[\frac{\partial D^{(p)}}{\partial C}\right]_{C=c_{n}} \\
\text { P=1 } C_{n+1}=C_{n}-\lambda_{1} y_{n} \cdot Z_{n}\left(1-\frac{R_{1}}{\left|Z_{n}\right|}\right) \\
\text { Where } \quad R_{1}=\frac{E\left(a_{m}^{2}\right)}{E\left(\left|a_{m}\right|\right)} \\
\mathrm{P}=2 \quad C_{n+1}=C_{n}-\lambda_{2} y_{n} \cdot Z_{n}\left(\left|Z_{n}\right|^{2}-R_{2}\right) \\
\text { Where } R_{2}=\frac{E\left(\left|a_{m}\right|^{4}\right)}{E\left(\left|a_{m}\right|^{2}\right)}
\end{gathered}
$$

where $\mathrm{c}$ is a vector of the tap weights, $\lambda 1$ and $\lambda 2$ are adjusting coefficients, yn is an input signal, $\mathrm{Zn}$ is an equalized signal (output of the equalizer), $\mathrm{Rp}$ is a positive number determined by the pattern of the constellation diagram, and am represents the exact coordinates on the constellation diagram. Simulations show that the convergence of tap weights is faster and more accurate when using the second order $(\mathrm{P}=2)$ cost function rather than the first order $(\mathrm{p}=1)$ cost function.

The adaptive multi-modulus equalization method for an adaptive equalizer proposed in the present invention comprises the following steps.

First, an input signal is stored and initial values for a plurality of equalizer coefficients (tap weights) are set. Then, the input signal is passed into the adaptive equalizer to generate an equalized signal. A cost is then calculated by substituting the equalized signal into a preset cost function. The cost is used to derive an adjustment amount for updating the tap weights of the adaptive equalizer. These steps are identical to the CMA algorithm in the prior to allow the tap weights to be updated for cost reduction. The adaptive equalizer switches to implementation of a multi-modulus algorithm (MMA) rather than the CMA algorithm when the cost is reduced to reach a first threshold. The MMA algorithm divides the constellation diagram into a plurality of regions, and the number of regions increases when switching to the subsequent stage. A key feature of the present invention is that there are stages with different numbers of regions. Modulus of each region for the subsequent stage is determined by statistical analysis of the equalized signal generated by the equalizer. The stored signal passes through the adaptive equalizer again when the equalizer switches to the MMA algorithm.

According to the MMA algorithm, the cost is now calculated regarding the equalized signal generated by the adaptive equalizer, as well as moduli of the corresponding regions. Then, an adjustment amount is derived by the cost, and is used to update the tap weights of the equalizer. The tap weights are continuously adjusted to reduce the cost. The adaptive equalizer switches to the subsequent stage when the cost reaches the threshold of the current stage. The thresholds are preset to switch to subsequent stages. The adaptive equalizer repeats the process of convergence by continuously updating the tap weights, reducing the cost, and switching to the subsequent stage until a desired value for the cost function output (cost) is reached. When the cost reaches the desired value, the number of regions and tap weights are fixed and the received signal is equalized thereby.

By switching to subsequent stages, the convergence rate is increased because the constellation diagram is divided into more reqions. A distance between any valid coordinate and its corresponding modulus is decreased by allowing more regions on the constellation diagram. Costs can be reduced more effectively by minimizing this distance, as explained later in detail. The adaptive MMA algorithm solves the problem of unacceptable convergence performance for QAM signals with large numbers of valid coordinates on the constellation diagram.

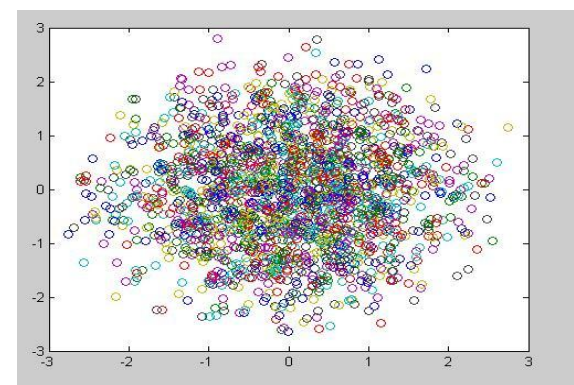

Figure 5. constellation diagram illustrating an output of an adaptive MMA equalizer when receiving QAM signal. 


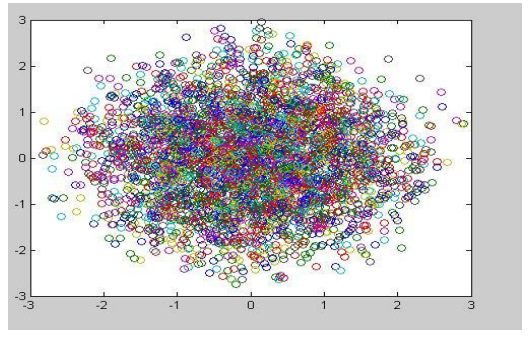

Figure 6 constellation diagram illustrating a signal received before passing through the decision feedback equalizer.

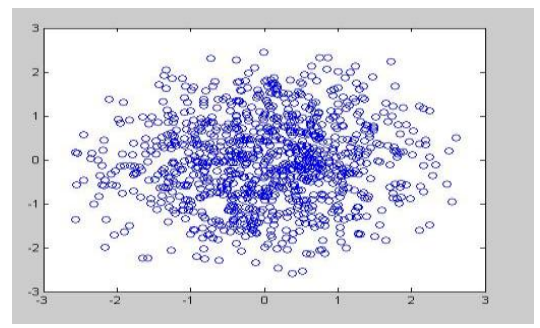

Figure 7 constellation diagram illustrating a signal received after passing through the decision feedback equalizer.

1. An adaptive multi-modulus equalization method for an equalizer, comprising the steps of:

.

storing an input signal;

generating initial values for a plurality of equalizer coefficients;

- calculating a constant modulus algorithm (CMA) cost function output according to a CMA with an equalized signal from the equalizer

- estimating a CMA adjustment amount for updating the equalizer coefficients according to the CMA cost function output;

determining modulus of each region for a multimodulus algorithm (MMA) by statistical analysis of the equalized signal;

switching the equalizer to use the MMA when the CMA cost function output reaches a first threshold, wherein the MMA comprises a plurality of stages determined by thresholds, and the number of regions increases in each subsequent stage; calculating a

MMA cost function output according to the MMA with the equalized signal and modulus of each region;

- estimating a MMA adjustment amount for updating the equalizer coefficients according to the MMA cost function output;

determining modulus of each region for the subsequent stage of the MMA by statistical analysis of the equalized signal;

switching the equalizer to the subsequent stage of the MMA when the MMA cost function output reaches the threshold corresponding to the current stage;

repeating the steps of calculating the MMA cost function output, determining modulus of each region, and switching the equalizer to the subsequent stage until the MMA cost function output reaching a preset value; and

fixing the number of regions and equalizer coefficients to equalize the input signal when the MMA cost function output has reached the preset value.

And also comprising the steps of:

phase recovering and non-linear transforming the equalized signal into a recovered signal; and inputting the recovered signal to a decision feedback equalizer. The CMA cost function output and the MMA cost function output are calculated by two second-order discrete cost functions. The constant modulus algorithm (CMA) is implemented by a steepest gradient descent algorithm. The multi modulus algorithm (MMA) is implemented by a steepestgradient descent algorithm.

6. An adaptive multi-modulus equalizing system, comprising: an equalizer, generating an equalized signal from an input signal according to equalizer coefficients;

a first coefficient generator, estimating a first adjustment amount by calculating a first cost function output according to a constant modulus algorithm (CMA);

a second coefficient generator, estimating a second adjustment amount by calculating a second cost function output according to a multi-modulus algorithm (MMA); and

a multiplexer connected to the equalizer, selecting either the first adjustment amount or the second adjustment amount with which to update the equalizer coefficients depending on a first threshold. The first coefficient generator calculates the first cost function output from the input signal and the equalized signal. Wherein the second coefficient generator calculates the second cost function output from the input signal, the equalized signal, and moduli obtained by statistical analysis of the equalized signal. and the number of moduli used to calculate the second cost function output is incremental when the second cost function output reached a second threshold.

10. An adaptive multi-modulus equalization method for an equalizer, comprising the steps of:

storing an input signal;

generating initial values for a plurality of equalizer coefficients;

calculating a constant modulus algorithm (CMA) cost function output according to a CMA with an equalized signal from the equalizer;

estimating a CMA adjustment amount for updating the equalizer coefficients according to the CMA cost function output; and

determining modulus of each region for a multi-modulus algorithm (MMA) by statistical analysis of the equalized signal.

\section{CONCLUSION}

In this paper we estimated blind channel equalization schemes particularly using multi modulus Algorithm (MMA) by considering constant Modulus Algorithm (CMA). We estimated a linear channel model driven by a QAM, PAM, BPSK source and adapt a Adaptive equalizer using CMA The adaptive MMA algorithm solves the problem of unacceptable convergence performance for QAM signals with large numbers of valid coordinates on the constellation diagram.

The object of the present paper is to provide an adaptive multi-modulus equalization method for a blind equalizer which overcomes the disadvantages of existing methods 
Drawbacks of the CMA algorithm include the performance of convergence for a Quadrature Amplitude Modulation (QAM) signal degrading with the number of valid coordinates on the constellation diagram. FIG. 2 shows the output of a CMA equalizer for a signal modulated by 896-QAM. There are 896 valid coordinates on the constellation diagram for a 896-QAM signal are overcome using MMA.

Reducing the cost also reduces the distance $\mathrm{d}$, and the cost can be reduced by implementing the steepest gradient descent method. The problem with the CMA algorithm is that $\mathrm{c}$ is a constant and cannot be reduced any further, hence the distance $\mathrm{d}$ becomes relatively small during reduction of the cost. The steepest gradient descent method cannot execute effectively and efficiently when the ratio of $d$ to the cost becomes too small.

\section{REFERENCES}

[1] Godard, D. N., "Self-recovering equalization and carrier tracking in two-dimensional data communication systems," IEEE Trans. on Communications.

[2] Fijalkow, I., C. E. Manlove, and C. R. Johnson, Jr., "Adaptive fractionally spaced blind CMA equalization: Excess MSE," IEEE Trans. on Signal Processing, Vol. 46, No. 1, 227-231, 1998.

[3] Ding, Z., R. A. Kennedy, B. D. O. Anderson and C. R. John- son, Jr., "Ill-convergence of godard blind equalizers in data communication systems," IEEE Trans. on Communications, Vol. 39.

[4] Johnson, Jr., C. R., S. Dasgupta, and W. A. Sethares, "Averaging analysis of local stability of a real constant modulus algorithm adaptive filter," IEEE

[5] Brown, D.R., P. B. Schniter, and C. R. Johnson, Jr.,

“ Computationally e cient blind equalization," 35th

Annual AllertonConference on Communication, Control, and Computing, September 1997.

[6] Casas, R. A., C. R. Johnson, Jr., R. A. Kennedy, Z.

Ding, and R. Malamut, "Blind adaptive decision feedback equalization: A class of channels resulting in illconvergence from a zero initialization," InternationalJournal on Adaptive Control and Signal Processing Special Issue on Adaptive Channel Equalization.

[7] Johnson, Jr., C. R. and B. D. O. Anderson, "Godard

blind equalizer error surface characteristics: White, zero-mean, binary source case," International Journal of Adaptive Control and Signal Processing, Vol. 9, 301324.

[8] Al-Kamali, F. S., M. I. Dessouky, B. M. Sallam, and F. E. Abd El-Samie, "Frequency domain interference cancellation ," Progress In Electromagnetics Research.

[9] Hanks and H.Zeng and Lang Tong "The Mse Perfromance Of Constant Modulus Algorithm".

Sridha vambu, Sergio Verdu, Rodney A. Kennedy and William Sethares "Convex Cost functions in Blind Equalization". IEEE trans on signal processing vol 42., no. 8. 1994. 